\title{
Development of dynamic impact cone for assessing induced partial saturation (IPS) as liquefaction mitigation technique
}

\author{
Fritz R. P. Nababan ${ }^{1, *}$ \\ ${ }^{1}$ Soil Mechanics Laboratory - ITB, Bandung, Indonesia
}

\begin{abstract}
Liquefaction of saturated loose sands during an earthquake has been a great concern for practicing earthquake engineer because of its damaging effects. Current liquefaction mitigation techniques used in practice are expensive. A new innovative mitigation technique which is cost effective was proposed by Yegian et al. [1] called "Induced Partial Saturation" (IPS). This method involves in generating gas bubbles within the pores of fully-saturated sand and transformed it to partially-saturated sand. This paper described the development of a dynamic impact cone to assess the effect of this IPS treatment on saturated loose sand specimens. This dynamic impact cone consists of a cone, rod, ruggedized pressure transducer, string pot, and DAQ. The cone which has pressure transducer incorporated at its tip was driven into fully and partially (treated) saturated sand specimens prepared in the laboratory. The excess pore pressures and penetrations depth per impact of the two specimens were compared. The tests results such as the excess pore pressure and the penetration indicate that the impact penetration on the fully-saturated specimens liquefied sand, but could not liquefy the partially-saturated sand. Therefore the concept and development of this dynamic impact cone has promising future applications.
\end{abstract}

\section{Introduction}

Liquefaction of saturated loose sands during earthquake event poses a great concern for practicing geotechnical earthquake engineer because tremendous damage it can cause. Researches on finding effective and cost effective liquefaction mitigation techniques are still undergoing in many part of the world. One promising new mitigation technique which is innovative, practical, and cost effective was proposed by Yegian et al. [1] called "Induced Partial Saturation" (IPS). This technique involves generating small amount of minute gas bubbles within the pores of initially fully-saturated sand, thus reducing the saturation degree and at the same time increasing the compressibility of the soil pores. Reduction of the pore's compressibility reduces the tendency of the pore fluid to generate excess pore water pressures. These generated gas bubbles in the pores are made by chemical reaction using environmentally friendly agent.

Small scale laboratory testing has shown the benefit of IPS as described in Yegian et al. [1] and EsellerBayat et al. [2],[3]. Most recently the benefit of IPS treatment was also observed and proven in large scale laminar box tests in NEES University of Buffalo facilities, these results were analyzed and presented by Nababan [4].

This paper describes idea and development of IPS verification technique to be used both in the laboratory and in the field. A verification method of IPS treatment which utilizes a dynamic impact cone was explored, which can verify the presence of gas bubbles in the pores by measuring the behavior of the specimens before and after the treatment. The cone is considered simple and practical to implement in the laboratory and in the field for specimens before and after IPS treatment. The detail of the developed cone is described herein as well as the typical results of the test.

\section{Dynamic Impact Cone}

The basic concept for IPS verification is comparing the excess pore pressure generation and the soil resistance if dynamic loading introduced to a specimen. A drop weight was chosen to generate dynamic impact load on the cone. The postulate is drop weight will impart large enough shear strains that it will liquefy the specimen and pore pressure transducer incorporated at the cone tip will measure a high excess pore pressures, enough to reduce the vertical stresses close to zero for the untreated specimen (i.e. liquefaction). It is expected that if the same cone is driven into the same specimen after being treated with IPS, the presence of gas bubbles in the pores will considerably reduce the generation of excess pore pressure. Comparing the difference of excess pore pressure records between the fully saturated and IPS treated specimens can then be considered as evidence of IPS treatment. Another hypothesis is that cone penetration due to impact of weight drop would be larger in the fully saturated (before IPS treatment) compare to that of partially saturated specimens (after IPS treatment).

\subsection{Prototype Cone}

A prototype of dynamic impact cone had been developed as shown in Figure 1 following. This cone

\footnotetext{
"Corresponding author: fritzuu@gmail.com
} 
consists of: an aluminum rod, aluminum cone, pressure transducer and gravity driven hammer system. The cone is attached to a $63.5 \mathrm{~cm}$ long hollow rod with diameter of $\varnothing 3.2 \mathrm{~cm}$. The cone is pointed at 60 degree, while the backside of the cone is a male thread to connect to the rod. The pressure transducer sits inside the cone facing towards the point and is fixed in placed by silicone. The tip of the transducer connected to a chamber with four holes on its side.

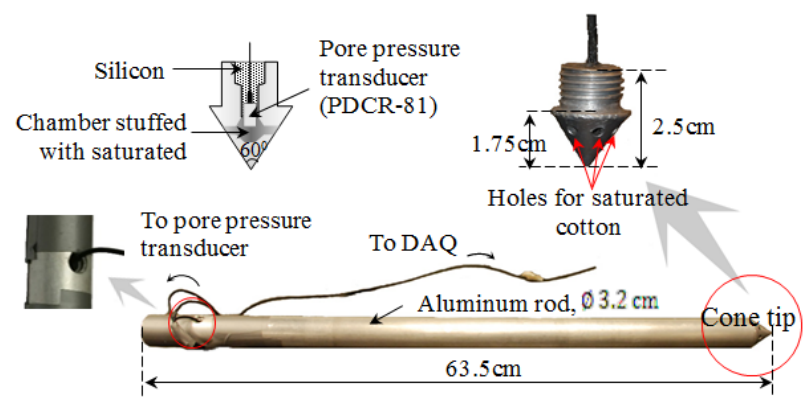

Fig. 1. Prototype cone.

In order to keep sand particles entering the chamber in which the transducer is fixed, saturated cotton is filled inside the chamber. The pore pressure transducer (PPT) used in this prototype is PDCR-81 (input 5V and output $0-100 \mathrm{mV}$, range pressure $0-5 \mathrm{psig}$ ), which contains a diaphragm with 4 strain gauges attached in full bridge configuration. This diaphragm is protected by a porous stone.

The specimens in general were prepared using Ottawa sand and de-aired water into a plastic barrel with known dimensions. Firstly, a measured quantity of de-aired water was placed in the barrel, and measured quantity dry Ottawa sand was rained into the barrel until no more water was left on top of the specimen. Knowing the weights and volumes of the sand, the water, and the final specimen height, the void ratio and the degree of saturation were computed using soil phase relations. The partial saturation specimen was prepared by mixing the chemical with de-aired water prior to sand pouring; the degree of saturation achieved for this specimen was about $70 \%$.

The summary of the tests done with this prototype cone in fully saturated as well as partially saturated specimens just proven the initial postulates. In the fully saturated specimen the impact caused the specimen to liquefy at the cone tip level which was verified by measured excess pore pressures at the tip. The measured excess pore pressure reached the effective overburden pressure at the same level. The measured excess pore pressure for IPS treated specimen were approximately $30 \%$ of the effective overburden pressure.

The limitations encountered of this cone were: 1) pressure transducer used for the cone is found not sturdy enough taking impact load as observed several of the transducers quitted during testing, 2) soils enter the chamber during testing as the stuffed saturated cotton is not enough to filter the soil.

\subsection{Developed Dynamic Impact Cone}

The dynamic impact cone should work for both laboratory and field applications and has to overcome the limitations of the prototype cone mentioned above. This cone is design to be used with drilling rod, E-rod, made of harden steel with diameter of 1.3 inch, small enough to be used in the laboratory and sturdy enough to be used in the field. The following figure shows detail of the cone developed.

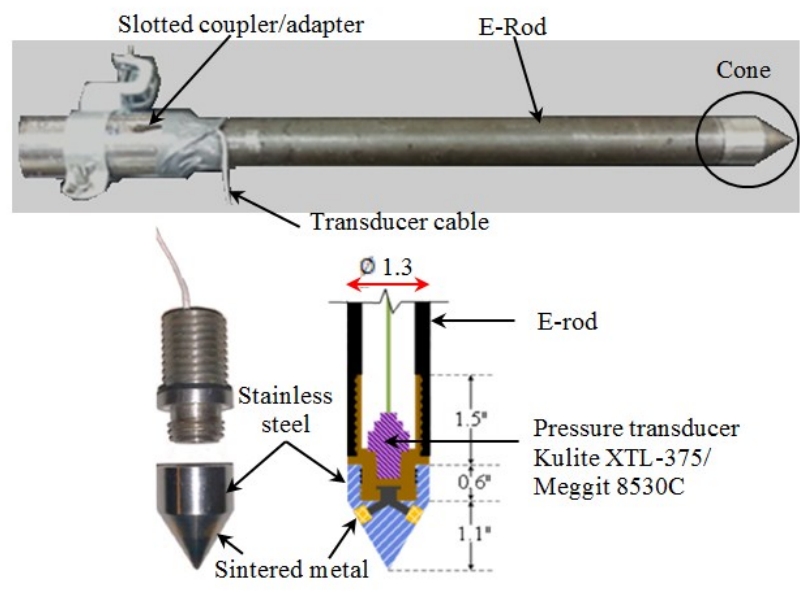

Fig. 2. Dynamic impact cone design used with drilling Erods (diam. 1.3 inch).

The developed dynamic impact cone consists of heavy duty ruggedized pressure transducer, saturation and calibration chamber, data acquisition system, and data processing software. Details of this cone are described in the following.

\subsubsection{Heavy Duty Pressure Transducer}

The pressure transducer needed for the system has to be able to withstand heavy beating and very high acceleration caused by the impact of the drop weight. The research on the suitable pressure transducer candidate came up with at least two types of proper commercial pressure transducer as shown in following figure 3. These are not pore pressure transducer but regular pressure transducers which have no porous stones. Instead of porous stones on their faces, they have stainless steel screens. This type of transducer can work well to measure liquid as well as gas pressures. The two miniature pressure transducers used for the cone were: Kulite XTL 375 with pressure range $0-25$ psia (absolute), and Meggit $8530 \mathrm{C}-100$ with pressure range of $0-100$ psia (absolute), reference pressure relative to the perfect vacuum. Both transducers were very sturdy and rugged, capable of working in extreme environments, and can withstand up to $10,000 \mathrm{~g}$ impact load and up to $1,000 \mathrm{~g}$ sinusoidal load. The output of both pressure transducers were in $\mathrm{mV}$ (millivolt), which required the use of the signal conditioner for data acquisition. 
(a)

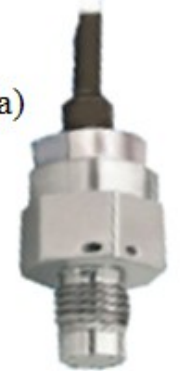

(b)

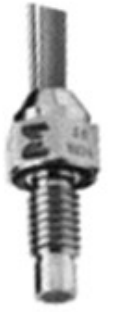

Fig. 3. Heavy duty ruggedized types of pressure transducers were explored (a) Kulite XTL 375 (b) Meggit 8530C-100.

\subsubsection{Saturation and Calibration Chamber}

The filter used to separate the cone chamber with surrounding soil is sintered metal, four sintered metal were installed at the tip of the cone as shown in figure 2 earlier. The chamber is filled with mixture of glycerin and water, this liquid act as medium in which the pressure from surrounding soil is transferred to transducer. It is essential to keep the sintered metal fully saturated with glycerin and water prior to each testing to avoid measurement delay and error. A chamber was made for saturation and calibration of the cone as illustrated in figure 4 the following. Air pump and vacuum were used for the chamber, air pressures were used for calibration and vacuum were used for cone saturation.

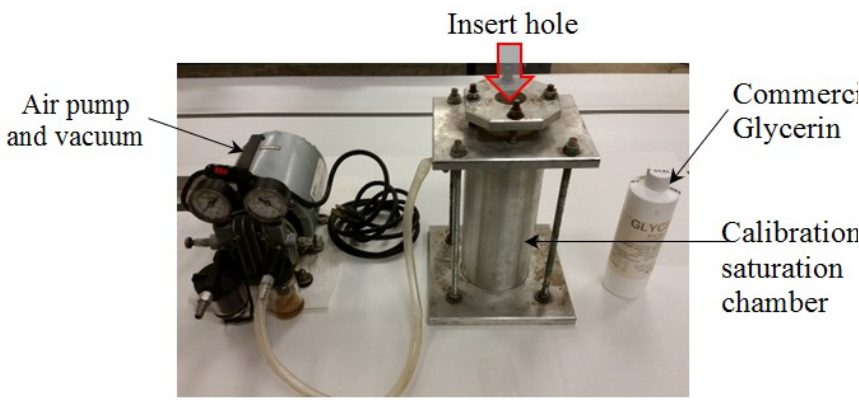

Fig. 4. Saturation and calibration chamber, air pump and vacuum, and glycerin.

The steps describe preparation and assemblies of the dynamic impact cone are as follow:

1- Saturation of the cone (sintered metal/ filter). The apparatus shown in figure 4 is utilized for this purpose. The chamber is filled halfway with $50 \%$ de-aired water and $50 \%$ glycerin and then the cone is placed inside and $20 \mathrm{psi}$ vacuum is applied for 6-8 hours.

2- The pressure transducer is then installed in its housing (see figure 2). The cable of the transducer is extended before placing and shrink wrap is used to make the connections watertight.

3- Assembling of transducer housing and the cone tip is done in a small container filled with saturation fluid, to ensure that the system remains saturated.
4- After the cone is assembled then it has to be recalibrated using the same chamber as shown in figure 4. Then the cone is inserted through the hole on the lid and air tight seal is created. The transducer cable is than connected to a portable data acquisition (PDAQ) and a laptop with installed data processing software, LabVIEW is used. Calibration is made by applying pump pressure and comparing with the transducer reading. Adjustments are made in the conditioner of the PDAQ system to ensure reliable transducer readings.

5- After calibration the AW rod segments are prepared to a length equal to an anticipated depth of a test. At this stage, the cone is ready for testing.

\subsubsection{Portable data acquisition (PDAQ) system for field application}

Portable data acquisition (PDAQ) system was developed alongside the dynamic impact cone as shown in figure 5 below. A case was used to house the PDAQ system, which is watertight, airtight, dustproof, crushproof.

The outside dimensions of Length 19-1/8", Width $15-7 / 16 "$ ", Depth $9 / 16 "$, and empty weight is about $4 \mathrm{~kg}$. Overall the components of the developed PDAQ system entail:

- Portable NI USB-6216 M

Powered by USB cable from the field laptop, this USB-NI 6216 has 16 analog input channels, sampling rate of $400 \mathrm{kS} / \mathrm{s}$, and powered by USB cable out from the laptop.

- Signal Conditioners

Signal conditioner used for the PDAQ is Ultra Slim Pak G448

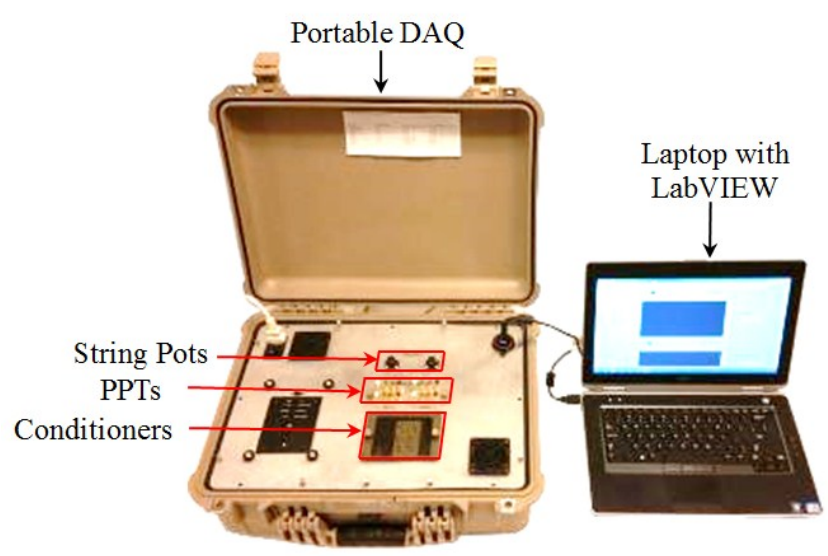

Fig. 5. Portable data acquisition (PDAQ) developed for dynamic cone testing.

- Auto recharge battery and Converter

A small and compact battery 24VDC was used to power the conditioners and the fan. $\mathrm{AC}$ to $\mathrm{DC}$ converter was also used to convert the voltage from $\mathrm{AC}$ wall to DC battery. 


\section{- Connectors}

Two types of connectors were made available in the front panel: first type is four units of 2 pin connector for the cone, and the second type is 2 units of shell-type connector for anticipated utilization of displacement transducer.

\subsubsection{Laptop and Data Processing Software}

Laptop is used with National Instrument (NI) data acquisition software, LabVIEW, for data recording and processing.

\section{Test and Results}

The developed dynamic impact cone then tested first in small scale test in the laboratory and followed by field testing. Test results of small scale specimen in the laboratory only presented in this paper.

\subsection{Specimen Preparation and Test Set Up}

The specimens were prepared using Ottawa sand and de-aired water in quite transparent barrel with a known dimensions. In brief, the preparation processes are as follow: 1) Measurement of quantity of de-aired water and Ottawa sand, 2) Placement of water inside the barrel, 3) Raining dry Ottawa sand into the barrel, 4) Reading the initial condition of the specimen. Given the weights and volumes of Ottawa sand, de-aired water, final specimen height, and free water height on top of the specimen, then the properties of specimen prepared such as void ratio, porosity, unit weight, and degree of saturation can be computed by mean of soil phase relations. Partially saturated specimen is prepared using a mixture of de-aired water and certain quantity of the environmental friendly chemical powder was used.

As stated earlier, proving the usefulness of dynamic impact cone in assessing IPS treated specimen, ones need to performed similar tests for both fully saturated and partially saturated specimens with the same initial relative density. Therefore, a standard set-up was made in the laboratory to test both specimens and to get comparable results. Figure 6 following illustrated the set up used for the test. In general, the set up entails of fixed frame, drop hammer with guide rod, height indicator, and the specimen barrel. This set up ensure that the impact energy delivered by drop hammer for every blows is identical and thus different responses of the specimens can be reasonably compared. The impact energy was maintained constant during the test by keeping the falling height equal for each blow. In laboratory test the drop weight used was $2.3 \mathrm{~kg}$ with falling height of $30 \mathrm{~cm}$.

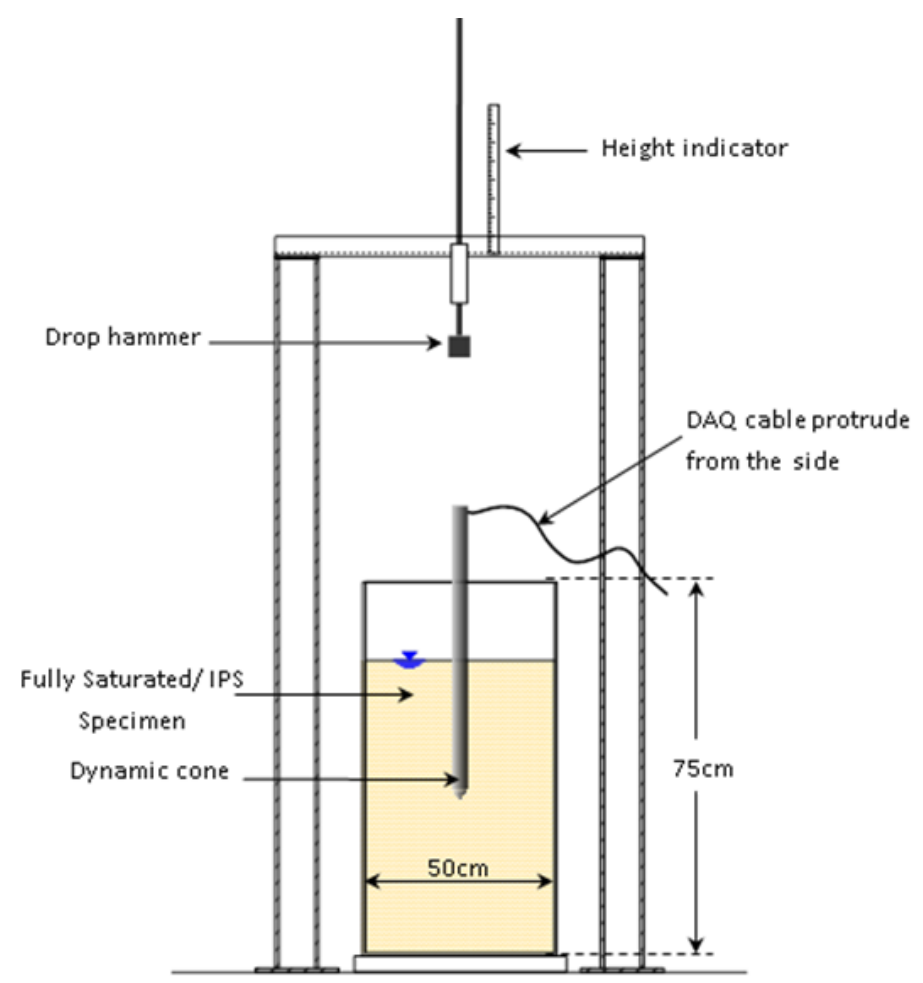

Fig. 6. Standard set up used in laboratory testing.

\subsection{Test Results}

Two tests of dynamic impact cone on fully and partially specimens were conducted and the results were compared. These specimens were prepared by method described previously with the same amount of sand and water. The resulting degree of saturation of the partially saturated sand was about $65 \%$. Both specimens had similar relative densities $\left(D_{r}\right)$ of about $50 \%$. The numbers on the photographs shown in figure 7 indicates top view of five test locations for each of the specimen where dynamic impact cone tests took place.

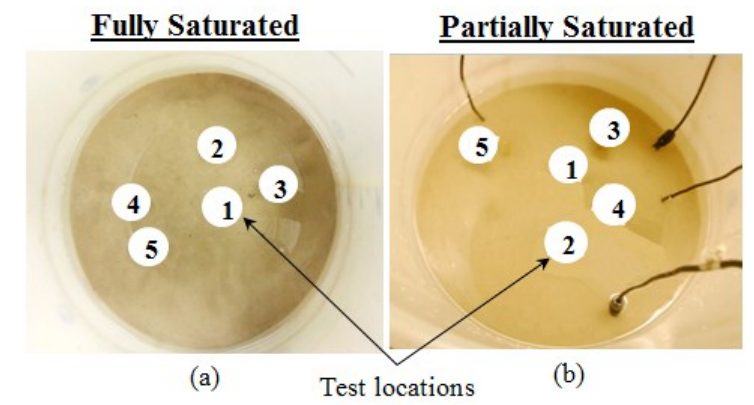

Fig. 7. Locations of cone tests in fully and partially saturated specimens.

Figure 8 shows the test results from the fully saturated sand specimen, when the cone was driven at locations of Tests 1 and 5. Initial relative density before Test 1 and 5 approximately are 50\% and 65\% consecutively. The spikes in the pressure transducer data show excess pore pressures developed during driving of the cone. The ratios of excess pore pressure 
and initial vertical effective stress were about $90 \%$, indicating that the cone almost liquefied the fully saturated sand specimen. While figure 9 shows results from testing the partially saturated sand specimen at location Test 1 and 2, where with $1 \%$ weight of environmentally friendly chemical. The initial relative density of the partially saturated specimen Test 1 and 2 were approximately the same with the fully saturated specimen test stated above, $50 \%$ and $65 \%$. It is noted that the maximum excess pore pressure generated by driving of the dynamic impact cone in the partially saturated specimen was about $35 \%$, this was significantly smaller than $90 \%$ recorded in the fully saturated specimen.
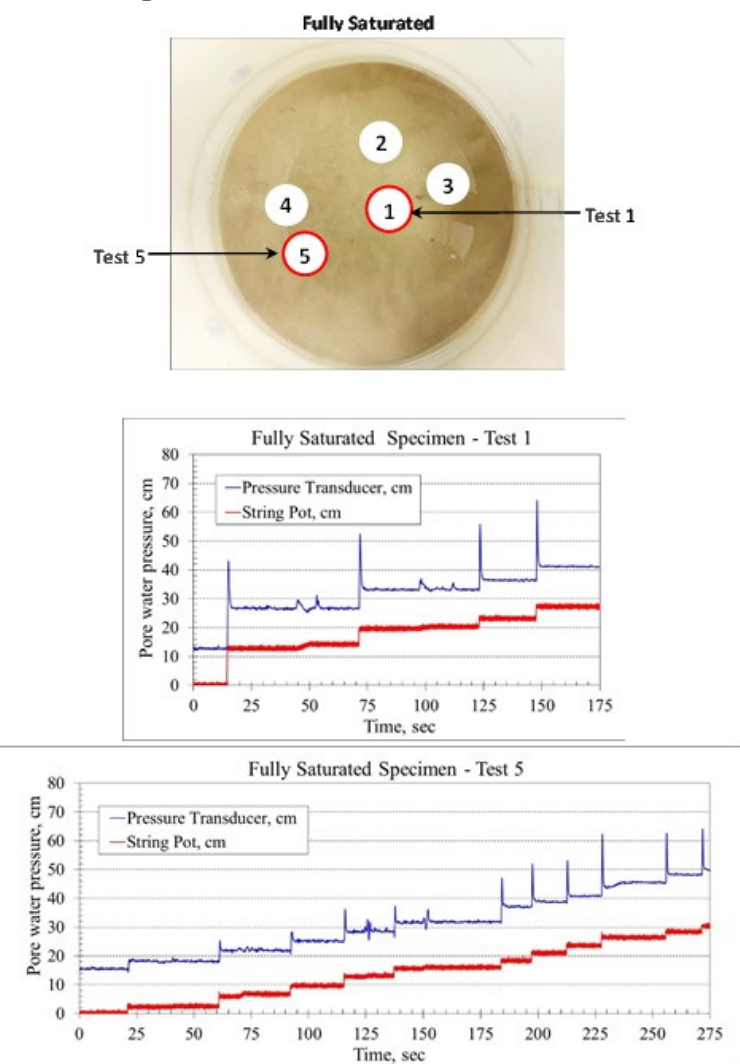

Fig. 8. Typical results of fully saturated specimen, Tests 1 and 5 .

Again these results demonstrated that dynamic impact cone can picked up the behavior differences of fully saturated and partially saturated specimens and therefore verify the effectiveness of IPS technique.

Another measured parameter from the tests that were useful in confirming the presence of gas bubbles and the effectiveness of IPS treatment is the penetration per blow of the cone. The initial postulate that the amount of penetration per blow of IPS treated specimen should be lower than fully saturated specimen was again confirmed as illustrated by figure 10. This figure shows that in order to penetrate approximately $25 \mathrm{~cm}$ of the prepared specimens, it took 4 blows for fully saturated specimen and 17 blows for partially saturated specimen. This behavior indicated that the presence of gas bubbles in pores of IPS treated specimen reduced the tendency of excess pore pressure generation during impact thus increase its bearing capacity if compare to fully saturated specimen, this explains why IPS treated specimen can take more beating to achieved the same penetration as the fully saturated specimen.
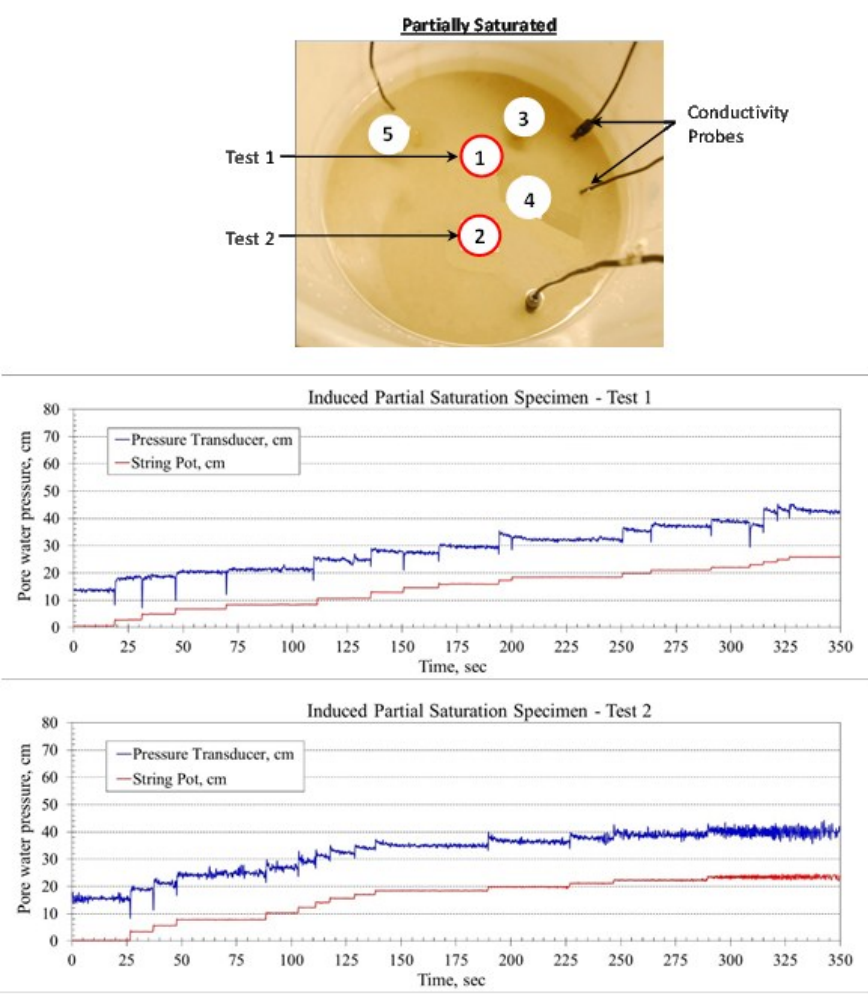

Fig. 9. Typical results of partially saturated specimen, Tests 1 and 2 .

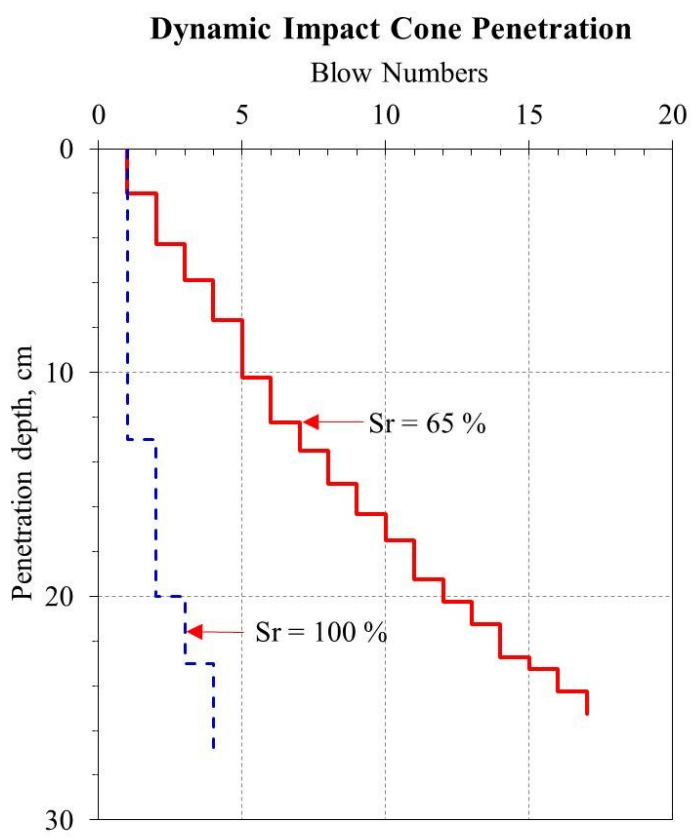

Fig. 10. Comparison of cone penetration of fully saturated and IPS treated specimen. 


\section{Conclusions and Recommendations}

The concept of using a dynamic impact cone to verify the presence and effect of partial saturation on a liquefaction potential sand specimen has been tested and had been proven effective in assessing the presence of gas bubbles in the pores of the specimen. There are two measured parameters that were used to verify IPS treatment: 1) excess pore pressure generated due to impact, and 2) amount of penetration per impact. The test results indicated that the presence of gas bubbles in the soil pores effectively reduced the tendency of the specimen to generate excess pore pressure and increase bearing capacity of the specimen relatively compare to fully saturated specimen.

It appears that laboratory test results shows promising future application of dynamic impact cone to be used for field application, mainly but not limited to assessing IPS treatment. Future research on this topic would be challenging at the same time promising.

Great appreciation goes to peoples that involved in this research to name a few: Hadi Kazemi, Seda Gokyer, Michael McNeil, Kurt Braun, and Mishac K. Yegian.

\section{References}

1. M. K. Yegian, E. Eseller-Bayat, A. Alshawabkeh, S. Ali, J. Geotech. Geoenviron. Eng., 133 (4), 372 (2007).

2. E. Esseler-Bayat, M. K. Yegian, A. Alshawabkeh, S. Gokyer, J. Geotech. Geoenviron. Eng., 139 (6), 863 (2013)

3. E. Esseler-Bayat, M. K. Yegian, A. Alshawabkeh, S. Gokyer, J. Geotech. Geoenviron. Eng., 139 (6), 872 (2013)

4. F. R. P. Nababan, Ph.D Diss., (2015) 